\title{
Acupuncture for the treatment of phantom limb syndrome in lower limb amputees: a randomised controlled feasibility study
}

\author{
Esmé G. Trevelyan*, Warren A. Turner, Lynn Summerfield-Mann and Nicola Robinson
}

\begin{abstract}
Background: Post amputation, the complication of phantom limb pain (PLP) is prevalent and difficult to manage. This study aimed to determine whether it was feasible and acceptable to undertake a definitive multicentred randomised controlled trial assessing the effectiveness of acupuncture for treating lower limb amputees with PLP.

Methods: A mixed-methods embedded design, including a randomised controlled trial and semistructured interviews, was undertaken. A total of 15 participants with PLP were randomly assigned to receive either eight pragmatic Traditional Chinese Medicine acupuncture treatments and usual care or usual care alone over 4 weeks. Outcome measures were completed at baseline, weekly throughout the study and at 1 month post completion of the study and included: a numerical pain-rating scale, the Short-Form McGill Pain Questionnaire 2, the EQ-5D-5 L, the Hospital Anxiety and Depression Scale, the Perceived Stress Scale 10-item, the Insomnia Severity Index, and the Patient Global Impression of Change. Post completion of the trial, participants in the acupuncture group were interviewed about their experience. Feasibility-specific data were also collected.
\end{abstract}

Results: Of 24 amputees meeting the study inclusion criteria, 15 agreed to participate (recruitment rate $62.50 \%$ ). Qualitatively, acupuncture was perceived to be beneficial and effective. Quantitatively, acupuncture demonstrated clinically meaningful change in average pain intensity (raw change $=2.69$ ) and worst pain intensity (raw change $=4.00$ ). Feasibility-specific data identified that before undertaking a definitive trial, recruitment, practitioner adherence to the acupuncture protocol, completion of outcome measures at 1 month follow-up and blinding should be addressed. Appropriate outcome measures were identified for use in a definitive trial. Data were generated for future sample size calculations (effect size 0.64). Allowing for a $20 \%$ dropout rate, a sample size of 85 participants per group would be needed in a future definitive trial.

Conclusions: A future definitive trial may be possible if the areas identified in this study are addressed. As acupuncture may be effective at treating PLP, and as this feasibility study suggests that a definitive trial may be possible, a multicentred trial with adequate sample size and blinding is now needed.

Trial registration: ClinicalTrials.gov Identifier: NCT02126436, registered on 4 September 2014.

Keywords: Phantom limb, Randomised controlled trial, Acupuncture, Amputation, Mixed-methods research

* Correspondence: trevelye@lsbu.ac.uk

School of Health and Social Care, London South Bank University, 103

Borough Road, London SE1 OAA, UK

(c) The Author(s). 2016 Open Access This article is distributed under the terms of the Creative Commons Attribution 4.0 International License (http://creativecommons.org/licenses/by/4.0/), which permits unrestricted use, distribution, and reproduction in any medium, provided you give appropriate credit to the original author(s) and the source, provide a link to the Creative Commons license, and indicate if changes were made. The Creative Commons Public Domain Dedication waiver (http://creativecommons.org/publicdomain/zero/1.0/) applies to the data made available in this article, unless otherwise stated. 


\section{Background}

Phantom limb pain (PLP) is defined as painful sensations in the missing portion of the amputated limb. It is neuropathic in nature and caused by a lesion of the somatosensory nervous system [1]. It may be chronic and has been found to influence individuals' subjective wellbeing, affecting both physical and mental components of quality of life [2].

Currently, PLP is not well-managed. A systematic review evaluating the use of preemptive analgesia found that only one case-controlled study supported using combined bupivacaine, diamorphine, and clonidine. Epidural and perineural infusions containing local anaesthetic with or without opiates were deemed only effective for treating acute perioperative pain [3]. A small randomised controlled trial found that intravenous ketamine could significantly reduce PLP during, and for 30 mins after, infusion [4]. However, a subsequent systematic review found it to be ineffective [5]. The most commonly used first-line treatment is gabapentin [6] but a systematic review found this to be beneficial for short-term analgesic efficacy only [7]. Many case studies report positively on the effectiveness of mirror therapy [8] but few randomised controlled trials have been completed and adverse effects have been reported.

Acupuncture has been found to be effective for treating a variety of chronic pain conditions [9] but little quality evidence is available on the use of acupuncture for PLP. A recent systematic review identified only two nonrandomised controlled trials [10] and 26 case studies [11]. Further research is needed to evaluate the effectiveness of acupuncture for treating PLP, but prior to a definitive trial a study is needed to inform on feasibility.

This study aimed to evaluate the feasibility and acceptability of completing a small randomised controlled trial in preparation for a definitive multicentred randomised controlled trial [12]. Objectives were to: (1) explore the feasibility of recruiting, randomising, and retaining participants, (2) evaluate the feasibility and acceptability of having a usual care control, (3) evaluate adherence/compliance and acceptability of acupuncture as an intervention, (4) evaluate the appropriateness of outcome measures, (5) identify appropriate primary and secondary outcome measures which could be used in future trials, (6) explore the perceived effectiveness of acupuncture for treating PLP, (7) generate data on effect size for use in future sample size calculations, and (8) inform the development of an appropriate and feasible protocol for use in a definitive multicentred randomised controlled trial.

\section{Methods}

A comparative effectiveness study using a mixed-methods embedded design, including a small randomised controlled trial incorporating semistructured interviews, was undertaken. The randomised controlled trial was unstratified, open, pragmatic, with two parallel arms, balanced randomisation and a usual care control. Interviews were cross-sectional. The study protocol has been published [12]. The trial was registered with ClinicalTrials.gov (NCT02126436). A Consolidated Standards of Reporting Trials (CONSORT) checklist is included within the article's additional files (Additional file 1, CONSORT 2010 checklist of information to include when reporting a randomised trial.pdf). Ethical approval was granted from the NRES Committee London - Bloomsbury (14/LO/0817) and London South Bank University; the trial commenced in October 2014 and closed 1 year later in October 2015.

Participants were recruited from an NHS inpatient amputee rehabilitation unit in London. All participants were provided with information and were required to consent orally and in writing. Participants were included if they were: (1) 18 years of age or above, (2) had full cognitive ability and were able to communicate in English, (3) had had traumatic or medical amputation of a lower limb (more than just toes), and (4) were currently experiencing worst PLP of $\geq 5$ on an 11-point verbal rating scale. Participants were excluded if they: (1) had congenital limb absence, (2) were medically unwell, (3) were pregnant, and (4) if acupuncture use was cautioned [13].

Participants were randomly allocated to either receive usual care and acupuncture or usual care alone. A usual care comparator was chosen as the study was undertaken under the Medical Research Council guidelines for developing and evaluating complex interventions. Usual care included pharmacological medical intervention, physiotherapy, and occupational therapy. Acupuncture was provided by an NHS clinic colocated in the same building by one British Acupuncture Council-registered acupuncture practitioner (BSc (Hons) Acupuncture) with more than 15 years of clinical experience. Acupuncture was delivered pragmatically under the Traditional Chinese Medicine (TCM) paradigm. A protocol developed prior to the study, using Delphi consensus methodology was used to provide guidelines [14] and included:

- Using a combination of body and auricular acupuncture

- Treating the contralateral limb and possibly the ipsilateral limb

- Including auricular acupuncture points such as the Shen Men and the sympathetic and points corresponding to the lower limb

- Depending on the health of the tissue and the individual participant, needling around the stump

- Mirroring local and distal points by needling the opposite limb

- Including points on the lower back (taking a segmental approach to dermatomal pain) 
- Including points such as LI4 + LR3, LR3, GV20, SP10, and also specified points according to participants' specific symptoms

- Attempting to obtain deqi

- Retaining needles for 20-30 min

Treatment could include electroacupuncture or other adjunctive interventions including cupping, exercises, and lifestyle advice. All participants in the acupuncture group were allocated eight 1-h sessions (twice weekly for 4 weeks).

Outcome measures were completed at baseline, weekly for the duration of the trial and 1 month post completion of the study. The primary outcome measure was an 11-point numerical rating scale (NRS) capturing average PLP over the past week, using the anchors 0 meaning 'no pain' and 10 meaning 'pain as bad as you can imagine' [15]. Secondary outcome measures included; NRS capturing worst PLP over the past week, the Short Form McGill Pain Questionnaire 2 (SF-MPQ-2) [16], the EuroQol-5 dimensions, 5 levels (EQ-5D-5 L) questionnaire [17], the Hospital Anxiety and Depression Scale (HADS) [18], the Perceived Stress Scale 10-item (PSS-10) [19], the Insomnia Severity Index (ISI) [20], and a 7-point Patient Global Impression of Change (PGIC) scale ranging from 1 meaning 'no change' to 7 meaning 'a great deal better'. Phrasing of the PGIC question was similar to the phrasing used by Hurst and Bolton [21] and stated 'since being enrolled in this study how would you describe the change (if any) in activity limitations, symptoms, emotion, and overall quality of life in relation to your phantom limb pain?' Feasibility-specific data were collected (Table 4) and post completion of the study, participants in the acupuncture group were interviewed. Interviews were conducted by the researcher who enrolled participants and collected outcome measures. Interviews were semistructured, audio-recorded, followed a topic guide and were transcribed verbatim.

No sample size calculation was undertaken but a sample of 20 was deemed adequate to inform on feasibility [22]. Interim safety and effectiveness were not formally evaluated but data were collected through participant interviews. Randomisation and allocation concealment was undertaken by a researcher not involved in the study using a computer-generated random numbers table. Randomisation was unstratified and balanced using a block size of 4 and allocation concealment was implemented using sequentially numbered opaque envelopes which were only opened once participants had been enrolled. The researcher collecting outcome measures and analysing the data enrolled the participants and was blinded to their allocation. Participants and acupuncture practitioners were not blinded.

Quantitative data analysis used an intention-to-treat approach and missing data were imputed using last observation carried forward. The intervention was discontinued after week 4 and this was chosen as the primary endpoint of the study (day 28). As this was a feasibility study no significance tests were performed and no hypothesis testing is reported. Raw change, the difference between mean baseline and subsequent scores was calculated for the NRS and considered meaningful/ clinically significant when $\geq 1.80$ [23]. Cohen's $d$ effect size was calculated using the calculation:

$$
d=M_{1}-M_{2} / \sigma
$$

where $M=$ mean and $\sigma=$ standard deviation, pooled using Cohen's criteria: 0.2, small effect; 0.5, medium effect; and 0.8, large effect [24]. Framework Analysis [25] was used to analyse qualitative data. Specific steps were followed during data analysis including: familiarisation, coding, identifying an analytical framework, indexing, charting, and mapping/interpretation. All codes and themes were developed inductively during analysis of the data. Inferences were drawn from analysis of qualitative and quantitative findings. Meta-inferences were drawn through combining qualitative and quantitative findings using side-by-side comparison [26].

Using effect size data generated from this study and taking the assumption that a future study would: (1) use an 11-point NRS measuring average pain over the last week, (2) have normally distributed data, (3) use a two-tailed independent samples $T$ test to compare acupuncture versus usual care, and (4) set power and level of significance/ $\alpha$-level at 0.8 and 0.05 , respectively, enabling a sample size for a future definitive trial to be calculated [27]:

$$
\frac{n=2\left(Z_{\mathrm{a}}+Z_{1-\beta}\right)^{2 \sigma 2}}{\Delta^{2}}
$$

where, $Z_{a}=1.96$ [27], $Z_{1-\beta}=0.8416$ [27], $\sigma=$ standard deviation (estimated) and $\Delta=$ estimated effect size.

\section{Results}

A total of 36 lower limb amputees were identified of whom 12 were ineligible. Of those eligible 9 refused to participate. A total of 15 participants were enrolled and their data were analysed within their originally assigned groups. Before the primary endpoint two were withdrawn due to being medically unwell and one dropped out having been randomised to usual care. A total of 12 participants completed outcomes at day 28, the primary endpoint of the study (seven in the acupuncture group and five in the usual care group). A total of 10 participants did not complete the 1-month follow-up questionnaire and 2 participants refused to be interviewed at the end of the study (Fig. 1).

Demographic details are presented in Table 1. Between groups there were differences in gender. In the acupuncture group six were men and in the usual care group five 


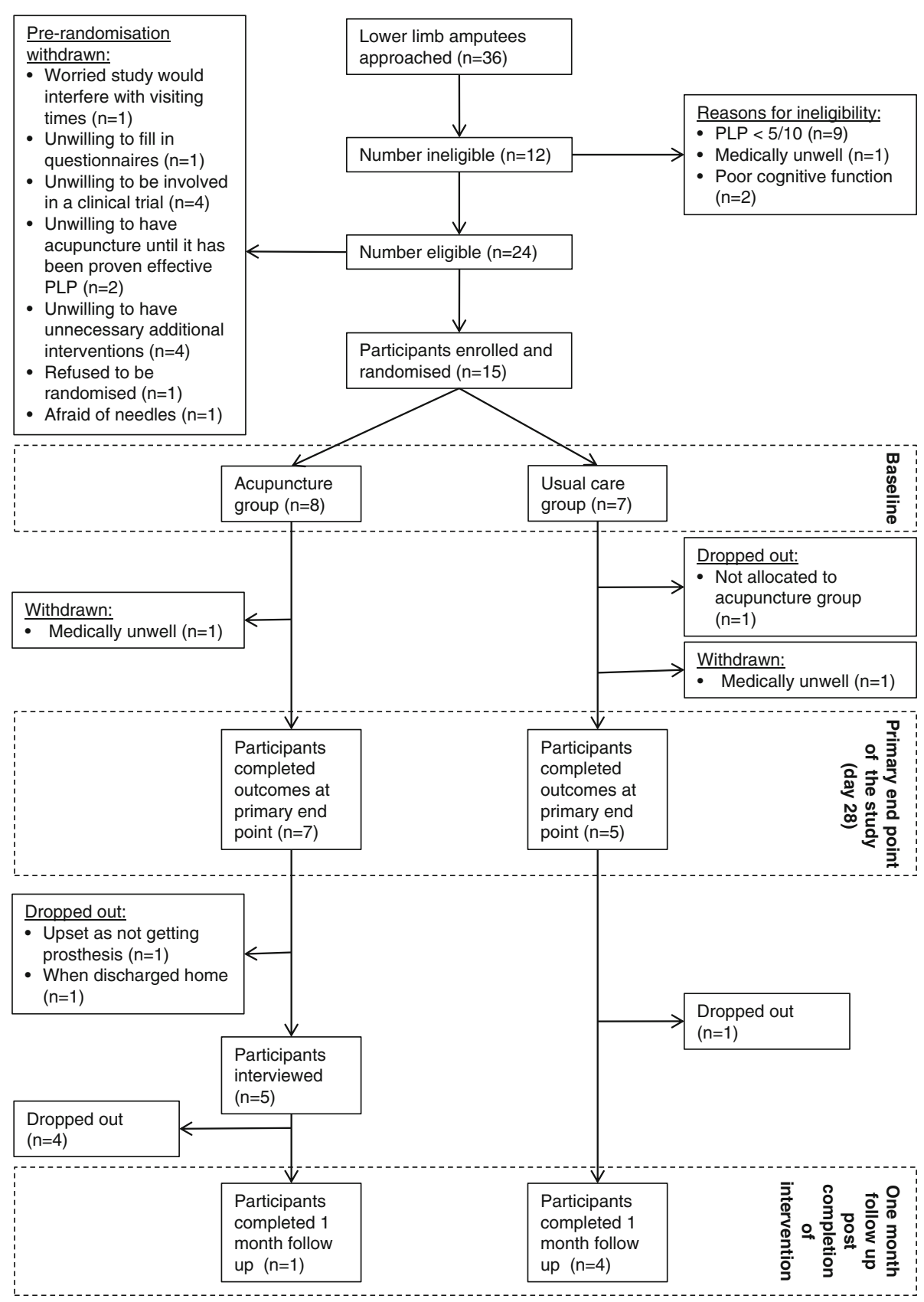

Fig. 1 Participant flow through the trial

were women. In the acupuncture group the majority of participants were below-knee amputees, whereas in the usual care group the majority were above-knee amputees. Baseline primary and secondary outcome measure scores were similar between groups.

\section{Quantitative findings}

In the acupuncture group mean average pain decreased from 5.44 to 2.75 and in the usual care group from 5.43 to 4.43 (Fig. 2). In the acupuncture group decrease in average pain was found to be clinically meaningful (raw change $=2.69$ ), but not in the usual care group (raw change $=1.00$ ). At day 28 , a medium effect was found between groups $(d=0.64)$.

In the acupuncture group decrease in mean worst pain was found to be clinically meaningful (raw change $=4.00$ ) but this was not so in the usual care group (raw change $=1.00$ ). The SF-MPQ-2 identified a small effect between groups at day $28(d=0.46)$. Mean HADS anxiety and depression scores were normal throughout 
Table 1 Participant demographics

\begin{tabular}{|c|c|c|}
\hline Participant demographics & $\begin{array}{l}\text { Acupuncture group } \\
(n=8)\end{array}$ & $\begin{array}{l}\text { Control group } \\
(n=7)\end{array}$ \\
\hline Age mean ( $\pm 95 \% \mathrm{Cl})$ & $51.63(40.38-62.87)$ & $55.71(40.17-71.26)$ \\
\hline \multicolumn{3}{|l|}{ Gender $n(\%)$} \\
\hline Male & $6(75.00)$ & $2(28.57)$ \\
\hline Female & $2(25.00)$ & $5(71.43)$ \\
\hline \multicolumn{3}{|l|}{ Ethnicity $n(\%)$} \\
\hline White British & $7(87.50)$ & $4(57.14)$ \\
\hline Black Caribbean & $1(12.50)$ & $1(14.29)$ \\
\hline Black African & $0(0.00)$ & $1(14.29)$ \\
\hline White other & $0(0.00)$ & $1(14.29)$ \\
\hline \multicolumn{3}{|l|}{ Employment status n (\%) } \\
\hline Student & $0(0.00)$ & $1(14.29)$ \\
\hline Unemployed & $1(12.50)$ & $0(0.00)$ \\
\hline Sick leave & $5(62.50)$ & $3(42.86)$ \\
\hline Retired & $2(25.00)$ & $3(42.86)$ \\
\hline $\begin{array}{l}\text { Time since amputation in } \\
\text { days mean }( \pm 95 \% \mathrm{Cl})\end{array}$ & $25.63(18.43-32.85)$ & $29.43(13.12-45.74)$ \\
\hline \multicolumn{3}{|l|}{ Level of amputation $n(\%)$} \\
\hline Above-knee & $2(25.00)$ & $4(57.14)$ \\
\hline Below-knee & $6(75.00)$ & $3(42.86)$ \\
\hline \multicolumn{3}{|l|}{ Reason for amputation $n(\%)$} \\
\hline Vascular & $5(62.50)$ & $3(42.86)$ \\
\hline Trauma & $2(25.00)$ & $2(28.57)$ \\
\hline Infection & $0(0.00)$ & $1(14.29)$ \\
\hline Other & $1(12.50)$ & $1(14.29)$ \\
\hline \multicolumn{3}{|c|}{ History of past amputations? n (\%) } \\
\hline Yes & $2(25.00)$ & $1(14.29)$ \\
\hline No & $6(75.00)$ & $6(85.71)$ \\
\hline \multicolumn{3}{|l|}{ General health $n(\%)$} \\
\hline Diabetes I & $1(12.50)$ & $0(0.00)$ \\
\hline Diabetes II & $3(37.50)$ & $2(28.57)$ \\
\hline Cancer & $1(12.50)$ & $0(0.00)$ \\
\hline Osteoarthritis & $1(12.50)$ & $0(0.00)$ \\
\hline Epilepsy & $1(12.50)$ & $0(0.00)$ \\
\hline $\mathrm{Nil}$ & $1(12.50)$ & $5(71.43)$ \\
\hline \multicolumn{3}{|l|}{ Mobility level $n$ (\%) } \\
\hline Wheelchair user & $8(100.00)$ & $7(100.00)$ \\
\hline
\end{tabular}

$\mathrm{Cl}$ confidence interval

the study in both groups (score $\leq 7$ ). As with the HADS, little change was observed in PSS-10 scores over the course of the study. Both groups at baseline had subthreshold insomnia (ISI score 8-14) which improved by day 28 . Throughout the study EQ-5D-5 L scores were stable across dimensions. At the primary endpoint of the study the PGIC identified that participants in the acupuncture group rated themselves as 'better' whereas participants in the usual care group rated themselves as 'a little better'. The datasets supporting these findings are included in Table 2.

\section{Qualitative findings}

Six themes were identified through interviews with participants who received acupuncture (Table 3). Participants were initially sceptical and apprehensive about being involved in the trial, had low expectations of acupuncture and hoped to be randomised to usual care. However, these views changed; participants liked treatment (even if it was not physically needed) and found it relaxing. Electroacupuncture was considered beneficial and pleasant and receiving two treatments a week was considered acceptable though some participants found this tiring. Acupuncture was perceived to be effective at resolving or reducing PLP and other health problems and four to six treatments were needed for it to be effective. Acupuncture was not perceived to cause any adverse effects. The environment where the acupuncture was conducted was considered to affect the effectiveness of treatment.

Completing outcome measures was considered acceptable and relevant, but the SF-MPQ-2 included words which some participants did not understand. Length of time and frequency of questionnaire completion was acceptable with only one participant thinking that they were given too often. Overall, being involved in the study was considered a good experience and acupuncture was perceived to be beneficial. Participant quotes are included in Table 3.

\section{Feasibility-specific findings}

Recruitment was problematic, clinicians sometimes failed to identify suitable participants, the unit did not always run at full capacity and potential participants were often unwilling to be involved having just had a major amputation. Of those identified, 12 were ineligible for inclusion, mainly due to PLP being less than $5 / 10$ in intensity and of the remainder $n=24,62.50 \%$ consented to be enrolled. Randomisation worked well with only one participant dropping out due to being randomised to usual care and all participants were treated in the group they were allocated into. Those enrolled reported being happy to be randomised to either acupuncture or usual care. Blinding was unsuccessful, with both participants and practitioners unintentionally informing the researcher which group they had been allocated to.

Participant compliance to the protocol was good [14]. The four participant deviations were due to: tiredness, forgetting appointments, appointments coinciding with another medical appointment, and not wanting further treatment as PLP had resolved. Practitioner adherence to the protocol was poor and no participant received all 


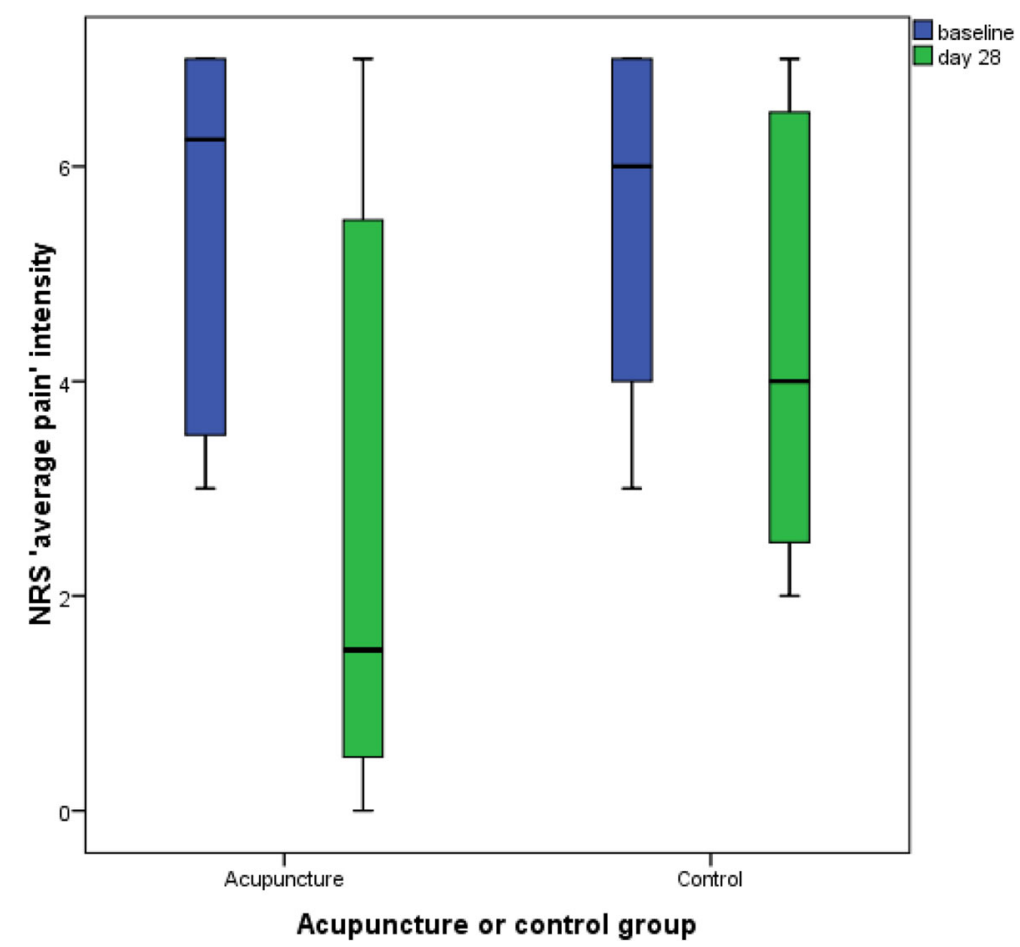

Fig. 2 Box plot of 'average pain' intensity at baseline and day 28

eight treatments (mean total number of treatments 5.14 (4.02-6.27)). Despite the protocol [14] advising using a combination of auricular and body acupuncture this was only given to one participant on two occasions. Both lower limbs were treated $66.67 \%$ of the time whereas for the contralateral limb this was only $8.33 \%$. Needle retention time and adverse events were not reported. For the dataset on acupuncture points used by practitioners in this feasibility study, see Additional file 2 (Acupuncture points used by practitioners during the feasibility study.pdf).

Outcome measures were identified which would be appropriate for a definitive trial. The NRS, SF-MPQ-2, and PGIC captured change. Baseline HADS scores were normal and little change was observed in the PSS-10 and the EQ-5D-5 L suggesting that these outcomes may be inappropriate. The ISI may not be appropriate in an inpatient setting as anecdotally noise and medication affected sleep. Retention of participants up until the primary endpoint of the study was good, but at 1-month follow-up was poor.

A sample size for a future trial was calculated corresponding to an effect size of 0.64 and pooled standard deviation of 1.36. A total of 71 per group (142 in total) would be needed. According to findings from this feasibility study, the follow-up rate at 4 weeks was $80 \%$. Therefore, considering a $20 \%$ dropout rate, 170 participants (85 per group) are recommended to detect a significant change in a two-armed, parallel-group randomised controlled trial comparing acupuncture and usual care as measured using an 11-point NRS measuring average pain at 4 weeks.

Using the criteria set a priori [12], as shown in Table 4 the study was found to be successful in relation to participants receiving the intended intervention, outcome measures being considered acceptable and appropriate and being completed at the primary endpoint of the study and the intervention being considered acceptable and appropriate for use in a definitive trial. The study was unsuccessful in relation to recruitment, practitioner adherence to the protocol, completion of outcome measures at 1-month follow-up and blinding.

\section{Discussion}

The study did not meet its target of recruiting at least 2 participants per month or 20 participants in total. This is not unusual and other studies have also reported recruitment as being slower or more difficult than expected [28]. It has been suggested that clinical staff have limited time to undertake research activities [29] and this may have influenced the identification of potential participants. A future trial would need to ensure that trial centres allocated adequate time and personnel. Potential participants should be provided with some education about the intervention as a brief introduction may make participants less sceptical and more willing to 
Table 2 Summary statistics at baseline and day 28 expressed as mean and between-group effect sizes

\begin{tabular}{|c|c|c|c|c|c|}
\hline Outcome measure & Group & $\begin{array}{l}\text { Baseline mean } \\
( \pm 95 \% \mathrm{Cl})\end{array}$ & $\begin{array}{l}\text { Between-group effect size } \\
\text { (Cohen's } d \text { ) }\end{array}$ & $\begin{array}{l}\text { Day } 28 \text { mean } \\
( \pm 95 \% \mathrm{Cl})\end{array}$ & $\begin{array}{l}\text { Between-group effect size } \\
\text { (Cohen's d) }\end{array}$ \\
\hline \multirow{2}{*}{$\begin{array}{l}\text { Number of participants who } \\
\text { provided complete data }\end{array}$} & Acupuncture & 8 & & 7 & \\
\hline & Usual care & 7 & & 5 & \\
\hline \multirow[t]{2}{*}{ NRS average pain } & Acupuncture & $5.44(3.90-6.98)$ & 0.00 & $2.75(0.31-5.19)$ & 0.64 \\
\hline & Usual care & $5.43(3.75-7.11)$ & & $4.43(2.37-6.49)$ & \\
\hline \multirow[t]{2}{*}{ NRS worst pain } & Acupuncture & $8.00(6.21-9.79)$ & 0.38 & $4.00(0.40-7.60)$ & 0.69 \\
\hline & Usual care & $7.29(5.80-8.77)$ & & $6.29(4.54-8.03)$ & \\
\hline \multirow[t]{2}{*}{ SF-MPQ-2 } & Acupuncture & $2.55(1.70-3.40)$ & 0.21 & $1.06(0.13-2.24)$ & 0.46 \\
\hline & Usual care & $2.85(1.22-4.47)$ & & $1.89(0.07-3.85)$ & \\
\hline \multirow[t]{2}{*}{ HADS anxiety } & Acupuncture & $6.38(2.75-10.00)$ & 0.29 & $5.25(1.97-8.53)$ & 0.10 \\
\hline & Usual care & $5.29(2.33-8.24)$ & & $4.86(1.38-8.34)$ & \\
\hline \multirow[t]{2}{*}{ HADS depression } & Acupuncture & $6.63(3.34-9.91)$ & 0.35 & $0.355 .75(1.35-10.15)$ & 0.12 \\
\hline & Usual care & $5.14(0.99-9.29$ & & $5.14(0.99-9.29)$ & \\
\hline \multirow[t]{2}{*}{ PSS-10 } & Acupuncture & 15.25 (10.90-19.60) & 0.31 & $11.63(5.43-17.82)$ & 0.48 \\
\hline & Usual care & $17.28(10.11-24.46)$ & & $15.57(7.35-23.79)$ & \\
\hline \multirow[t]{2}{*}{$|S|$} & Acupuncture & $13.50(5.96-21.04)$ & 0.49 & $8.50(1.65-15.35)$ & 0.14 \\
\hline & Usual care & $9.14(0.93-17.35)$ & & $7.42(0.61-14.24)$ & \\
\hline \multirow[t]{2}{*}{ EQ-5D-5 L mobility } & Acupuncture & $4.88(4.58-5.17)$ & 0.00 & $3.75(2.88-4.62)$ & 0.47 \\
\hline & Usual care & $4.88(4.58-5.17)$ & & $4.29(3.13-5.45)$ & \\
\hline \multirow[t]{2}{*}{ EQ-5D-5 L self care } & Acupuncture & $1.75(1.16-2.34)$ & 0.24 & $1.63(1.00-2.25)$ & 0.09 \\
\hline & Usual care & $1.57(0.84-2.30)$ & & $1.57(1.08-2.07)$ & \\
\hline \multirow[t]{2}{*}{ EQ-5D-5 L usual activities } & Acupuncture & $3.75(2.68-4.82)$ & 0.45 & $2.88(2.18-3.57)$ & 0.84 \\
\hline & Usual care & $4.29(3.26-5.31)$ & & $3.71(2.69-4.74)$ & \\
\hline \multirow[t]{2}{*}{ EQ-5D-5 L pain-discomfort } & Acupuncture & $3.50(2.87-4.13)$ & 1.24 & $2.88(2.18-3.57)$ & 0.44 \\
\hline & Usual care & $2.71(2.26-3.17)$ & & $2.57(2.08-3.07)$ & \\
\hline \multirow[t]{2}{*}{ EQ-5D-5 L anxiety/depression } & Acupuncture & $2.00(1.11-2.89)$ & 0.46 & $2.00(1.23-2.77)$ & 0.50 \\
\hline & Usual care & $1.57(0.84-2.30)$ & & $1.57(0.84-2.30)$ & \\
\hline \multirow[t]{2}{*}{ EQ-5D-5 $L$ health today } & Acupuncture & $63.13(46.41-79.84)$ & 0.21 & 74.63 (58.49-90.76) & 0.15 \\
\hline & Usual care & $67.14(50.29-84.00)$ & & 77.14 (63.05-91.23) & \\
\hline \multirow[t]{2}{*}{ PGIC } & Acupuncture & & & $5.71(4.23-7.20)$ & 1.27 \\
\hline & Usual care & & & $3.20(0.37-6.03)$ & \\
\hline
\end{tabular}

Cl confidence interval, EQ-5D-5 L EuroQol-5 dimensions, HADS Hospital Anxiety and Depression Scale, ISI Insomnia Severity Index, NRS numerical rating scale, PGIC Patient Global Impression of Change, PSS-10 Perceived Stress Scale 10-item, SF-MPQ-2 Short Form McGill Pain Questionnaire 2

consent. Recruitment could be enhanced by a multicentred approach. Intensity of PLP was a major barrier to recruitment. Although PLP can be severe, this may only be in approximately $30 \%$ of amputees [30, 31] explaining why this inclusion criterion excluded nine participants. Future studies may consider lowering or excluding the severity of this criterion.

The study did not meet its target of recruiting at least $70 \%$ of all eligible participants. However, this criterion was unrealistically high and $62.50 \%$ of all eligible participants were recruited. Other CAM studies report a lower participation rate [32] and studies evaluating the effectiveness of interventions for treating PLP also report a lower participation rate [33]. This study may not have met its target recruitment rate because it was set unrealistically high. Participation rate was good, suggesting that a future trial would be possible.

Amputees have often undergone extensive unpleasant interventions prior to amputation, and this may partly explain the reason for those refusing to consent. The study site may not have been optimal for recruiting due to it being a busy unit providing rehabilitation care for those at a key life point. Although, overall, recruitment was good, future studies may benefit by including amputees who are 
Table 3 Acupuncture group participant quotes from semistructured interviews

\begin{tabular}{|c|c|}
\hline Theme & Quote \\
\hline Scepticism and lack of expectations & $\begin{array}{l}\text { 'I was a bit worried about what it was all about. You said "acupuncture" and I said "I'm not keen on that" } \\
\text { And then I thought I'll try it, I'll try it out.' (Interviewee 1) } \\
\text { 'Didn't expect it to work. Very, very, very sceptical me, very. That's how ignorant I was... I didn't think it } \\
\text { would work, I thought it was all nonsense.' (Interviewee 4) }\end{array}$ \\
\hline Being treated & $\begin{array}{l}\text { 'It was so relaxing... and, um, I was just lying there and I could have quite easily gone to sleep! It was so } \\
\text { relaxing, so sort of peaceful.' (Interviewee 2) }\end{array}$ \\
\hline Changes in phantom limb pain & $\begin{array}{l}\text { 'I said "well, it's good. It's very good..." It works good and then last time she came she said "aren't you } \\
\text { going to have it?" I said "no, no, it's got rid of that pain that was down there".' (Interviewee 1) }\end{array}$ \\
\hline Factors affecting treatment & $\begin{array}{l}\text { 'She (the acupuncturist) looked after you well and I think that was a lot of it, her personality and the way } \\
\text { she treated you and everything.' (Interviewee 2) } \\
\text { 'I think because of the environment it was being done in and the timing more than anything, I think it } \\
\text { wasn't really a positive thing. It might have been a different story in another setting, if I had more time } \\
\text { around my schedule.' (Interviewee 3) }\end{array}$ \\
\hline Completing the outcome measures & $\begin{array}{l}\text { (Re the SF-MPQ-2) ' A couple of the wordings were a bit weird. I didn't get some of the words on the } \\
\text { describe the pain, gruelling and what was the other one? There were a couple of them I didn't } \\
\text { understand what these words meant.' (Interviewee 4) }\end{array}$ \\
\hline A good experience & $\begin{array}{l}\text { 'It's been very positive... it's been an extra benefit I would definitely say that... I couldn't criticise } \\
\text { anything to be perfectly honest.' (Interviewee 5) }\end{array}$ \\
\hline
\end{tabular}

SF-MPQ-2 Short Form McGill Pain Questionnaire 2

not in an inpatient unit receiving multiple interventions at a key life point and by making the proposed intervention less intensive.

Blinding was unsuccessful. A future study may benefit from clearly including information on the participant information sheet about the necessity of blinding and should ensure that the outcome measures used are reliable and objective. Additionally, a future trial could use duplicate assessments of outcomes and report the level of agreement between assessors [34]. Also, different data analysts to data collectors could be used.

Establishing acceptability and compliance to an intervention is vital because if the intervention is unacceptable and participants are not compliant, the study will fail. This study suggested that acupuncture and usual care were acceptable and participants were compliant with the protocol. Unlike usual care alone, acupuncture did appear to be clinically effective at reducing pain intensity and the findings suggested a 'meaningful change' [23]. This is in keeping with results from case studies [11] and nonrandomised controlled trials [10]. Clinically meaningful change is important as this is relevant to patient care. Across a diverse patient group a change of 1.74 on an 11-point NRS has been associated with 'much improved' and a change of 2.76 'very much improved' [35] suggesting that by the primary endpoint participants in the acupuncture group average pain was 'much improved' and worst pain was 'very much improved' but this was not so in the usual care group for either average or worst pain. In keeping with quantitative findings, qualitatively acupuncture was perceived to be effective at resolving symptoms. Findings from this study support the need for a definitive trial to determine effectiveness. As less than eight treatments may be effective this may be a more appropriate and cost-effective dosage. A usual care control should be used in future studies as it has the advantage of being safe (physicians make individualised treatment decisions about participant care) and, unlike efficacy trials, ensures that the intervention can claim to be superior to usual practice [36].

Unexpectedly, practitioners were found to not adhere to the acupuncture protocol. This lack of adherence may have been partly due to tensions between clinical and research workload [29] and also due to poor communication with the research team. This would need addressing before undertaking a future trial as lack of participants receiving the full intervention as intended could lead to reduced effectiveness, a decrease in study power and inappropriate conclusions [37]. Robiner [38] provides a table of adherence-enhancing strategies which could be used in a future trial, including: promoting collaboration and good communication between acupuncturists and research staff, providing feedback on adherence, promoting nonjudgemental discussion around adherence, and addressing adherence problems proactively.

Although adverse events were captured during semistructured interviews, practitioner compliance of capture of adverse effects was poor. This is not uncommon [39] but would need addressing before undertaking a definitive trial. Recommendations of capture of adverse events include capturing the frequency, incidence, timing, and severity of each event [40]. A future study may benefit from giving practitioners a log book designed to capture this information.

The study identified appropriate outcome measures which could be used in a future trial. However, as the SF-MPQ-2 included some terminology which was not understood, an alternative outcome measure may be 
Table 4 Success of feasibility study

\begin{tabular}{|c|c|c|}
\hline A priori criteria & Findings & $\begin{array}{l}\text { Objective met? } \\
\text { (yes/no) }\end{array}$ \\
\hline $\begin{array}{l}\text { Recruitment rate was } \geq 2 \text { participants per month fitting the } \\
\text { eligibility criteria }\end{array}$ & Recruitment rate was 1.36 eligible participants per month & No \\
\hline The study recruited $\geq 70 \%$ of all eligible potential participants & $62.50 \%$ of all eligible participants were recruited & No \\
\hline $\begin{array}{l}\text { Of the participants recruited to acupuncture group } \geq 90 \% \\
\text { received their first acupuncture treatment within } 1 \text { week of }\end{array}$ & $\begin{array}{l}\text { All participants received their first acupuncture treatment } \\
\text { within } 1 \text { week of recruitment }\end{array}$ & Yes \\
\hline
\end{tabular}
recruitment

After randomisation and allocation $\geq 90 \%$ of participants
received treatment as initially intended

Of the participants recruited to acupuncture group $\geq 80 \%$ received all 8 acupuncture treatments

Of the participants recruited to usual care group $\leq 10 \%$ dropped out of the study

At the primary endpoint of the study outcome measures were completed by $\geq 90 \%$ of participants

At 1 month after completion of the study, outcome measures were completed by $\geq 60 \%$ of participants

Qualitative data identified that outcome measures were acceptable and appropriate, that questionnaires and rating scales were easy to complete and that outcome measures could be identified for use in a definitive trial

Qualitative data implied that acupuncture was an acceptable and effective intervention for treating PLP with or without other secondary symptoms

Data were collected on the primary outcome measure (NRS) and effect size was calculated to inform a sample size calculation for a larger trial

Qualitative and quantitative data implied that the acupuncture protocol used in the feasibility study was appropriate for use in a definitive multicentred randomised controlled trial

The researcher was not aware which group participants had been enrolled to $100 \%$ of the time

\section{All participants received treatment as intended and the study \\ Yes} protocol was considered acceptable

No participants received all 8 treatments (mean total number of treatments 5.14 (4.02-6.27))

One participant (14.29\%) of participants dropped out of the usual care group

$100 \%$ of participants still enrolled on the study completed all outcome measures by the primary endpoint of the study

Outcome measures were completed by 5 participants (33.33\%)

Outcome measures were considered acceptable, appropriate and easy to complete. The HADS, PSS-10, EQ-5D-5 L, and ISI may not be appropriate for use in a definitive trial

Acupuncture/electroacupuncture was considered acceptable. Acupuncture was perceived to be effective at treating both PLP and other secondary complaints

Considering a $20 \%$ dropout rate, 170 participants are recommended to be recruited to detect a significant change in a two-armed, parallel-group randomised controlled trial comparing usual care and acupuncture as measured using an 11 -point NRS measuring average pain at 4 weeks

Participants did not drop out of the acupuncture group suggesting that it was acceptable. Participants' symptoms generally improved over 6 treatments suggesting that 8 treatments was adequate. Acupuncture and electroacupuncture were considered acceptable, effective, and relaxing

Blinding was not successful and the researcher knew through both participants and clinical staff at the amputee unit their group allocation
No

No

Yes

No

Yes

Yes

Yes

Yes

No

EQ-5D-5 L EuroQol-5 dimensions, HADS Hospital Anxiety and Depression Scale, ISI Insomnia Severity Index, NRS numerical rating scale, PSS-10 Perceived Stress Scale 10-item, SF-MPQ-2 Short Form McGill Pain Questionnaire 2

more appropriate such as the neuropathic pain scale, the neuropathic pain symptom inventory, or the Pain Quality Assessment Scale [41, 42]. Although participants adhered to completing outcome measures, this was not sustained post the primary endpoint of the study. This lack of long-term retention needs addressing as poor retention has implications for statistical power and the internal and external validity of a study [43]. Strategies could be implemented such as including a follow-up contact, prenotification reminders, and mentioning an obligation to respond [44]. In randomised controlled trials offering and giving small monetary incentives has been found to be successful in improving response [43]. However, lower limb amputees tend to be a frail population and long-term survival post amputation is poor. By 1 year post amputation almost half (44\%) of lower limb amputees will have died and by 5 years $77 \%$ [45]. Additionally, major amputations are associated with high morbidity and complication rates. This would need to be taken into consideration when designing a definitive trial.

\section{Limitations}

This study did not consider the effect of attention on symptoms and did not include a control that mimicked the theoretically inactive elements but not the active elements of acupuncture. Further research needs to be carried out to identify optimal dosage, which aspects of acupuncture intervention causes change and whether environmental factors affect outcomes. The study did not recruit the number of participants it initially aimed to recruit and the quantitative findings reported in this 
study should be interpreted with caution. Only one practitioner was involved in this study and as differences in effectiveness are known to occur with different practitioners [46] future studies would benefit from the use of multiple practitioners. Practitioners did not adhere to the acupuncture protocol and participants were not offered eight treatments, making it difficult to determine the effectiveness of the protocol. Two participants in the acupuncture group were not interviewed and data saturation of qualitative data cannot be assumed.

\section{Conclusions}

The study provides novel data on the feasibility of conducting a randomised controlled trial to establish the effectiveness of acupuncture for treating lower limb amputees with PLP. The study identified that acupuncture may cause clinically meaningful change. The protocol used in this study was acceptable and data on effect size were generated allowing for a sample size calculation. Areas which would need addressing prior to undertaking a definitive trial were identified.

\section{Additional files}

Additional file 1: CONSORT 2010 checklist of information to include when reporting a randomised trial. (PDF $144 \mathrm{~kb}$ )

Additional file 2: Acupuncture points used by practitioners during the feasibility study. (PDF $84 \mathrm{~kb}$ )

\section{Abbreviations}

EQ-5D-5 L: EuroQol-5 dimensions, 5 levels questionnaire; HADS: Hospital Anxiety and Depression Scale; ISI: Insomnia Severity Index; PGIC: Patient Global Impression of Change; PLP: Phantom limb pain; PSS-10: Perceived Stress Scale 10-item; SF-MPQ-2: Short Form McGill Pain Questionnaire 2; TCM: Traditional Chinese medicine;

\section{Acknowledgements}

The study was possible thanks to clinical staff at the Amputee Rehabilitation Unit, especially Jodie Georgiou and Dr. Carmen Calin, acupuncturists at the Gateway Acupuncture Clinic, especially Dominique Joire, and funding obtained from Guy's and St. Thomas' Charity and the Association of Traditional Chinese Medicine and Acupuncture UK.

\section{Funding}

Guy's and St Thomas' Charity and the Association of Traditional Chinese Medicine and Acupuncture UK.

\section{Availability of data and materials}

The datasets during and/or analysed during the current study are available from the corresponding author on reasonable request.

\section{Authors' contributions}

All authors were involved in the design of the study and analysis and interpretation of data. All authors reviewed and commented on the manuscript and gave final approval of the version to be published.

\section{Authors' information}

EGT is both a chartered physiotherapist and an acupuncturist and is currently completing a PhD at London South Bank University. NR completed a PhD in 1976, has been a licensed Traditional Chinese Medicine acupuncturist since 1982, and is a professor of Chinese medicine and integrated health at London South Bank University. WAT is a podiatrist; he completed a PhD in 2001 and is
Pro-Vice-chancellor and Dean of the School of Health and Social Care at London South Bank University. LSM completed a PhD in 2010 and is an associate professor and preregistration lead in the Department of Allied Health Sciences at London South Bank University.

\section{Competing interests}

The authors declare that they have no competing interests.

\section{Consent for publication}

Not applicable.

\section{Ethics approval and consent to participate}

Ethical approval was granted from NRES Committee London - Bloomsbury (14/LO/0817) and London South Bank University. All participants consented to participate both orally and in writing.

Received: 17 May 2016 Accepted: 4 October 2016

Published online: 25 October 2016

\section{References}

1. Flor H. Phantom-limb pain: Characteristics, causes, and treatment. Lancet Neurol. 2002;1(3):182-9.

2. Sinha R, van den Heuvel WJ, Arokiasamy P. Factors affecting quality of life in lower limb amputees. Prosthet Orthot Int. 2011;35(1):90-6.

3. Ypsilantis $E$, Tang TY. Pre-emptive analgesia for chronic limb pain after amputation for peripheral vascular disease: a systematic review. Ann Vasc Surg. 2010;24(8):1139-46.

4. Nikolajsen L, Hansen CL, Nielsen J, Keller J, Arendt-Nielsen L, Jensen TS. The effect of ketamine on phantom pain: a central neuropathic disorder maintained by peripheral input. Pain. 1996;67(1):69-77.

5. Humble SR, Dalton AJ, Li L. A systematic review of therapeutic interventions to reduce acute and chronic post-surgical pain after amputation, thoracotomy or mastectomy. Eur J Pain. 2015;19(4):451-65.

6. Hall GC, Morant SV, Carroll D, Gabriel ZL, McQuay HJ. An observational descriptive study of the epidemiology and treatment of neuropathic pain in a UK general population. BMC Fam Pract. 2013; doi:10.1186/1471-2296-14-28.

7. Alviar MJ, Hale T, Dungca M. Pharmacologic interventions for treating phantom limb pain. Cochrane Database Syst Rev. 2011; doi: 10.1002/ 14651858.CD006380.pub2.

8. Foell J, Bekrater-Bodmann R, Diers M, Flor H. Mirror therapy for phantom limb pain: brain changes and the role of body representation. Eur J Pain. 2014;18(5):729-39.

9. Vickers AJ, Cronin AM, Maschino AC, Lewith G, MacPherson H, Foster NE, et al. Acupuncture for chronic pain: individual patient data meta-analysis. Arch Intern Med. 2012;172(19):1444-53.

10. Hu X, Trevelyan E, Yang G, Lee MS, Lorenc A, Liu J, et al. The effectiveness of acupuncture/TENS for phantom limb syndrome. I: a systematic review of controlled clinical trials. Eur J Integr Med. 2014;6(3):355-64.

11. Hu X, Trevelyan E, Yang G, Lee MS, Lorenc A, Liu J, et al. The effectiveness of acupuncture or TENS for phantom limb syndrome. Il: a narrative review of case studies. Eur J Integr Med. 2014;6(3):365-81.

12. Trevelyan EG, Turner WA, Robinson N. Acupuncture for the treatment of phantom limb pain in lower limb amputees: study protocol for a randomized controlled feasibility trial. Trials. 2015;16(158): doi:10.1186/ s13063-015-0668-3.

13. British Acupuncture Council. Guide to safe practice. London: The British Acupuncture Council; 2010.

14. Trevelyan EG, Turner WA, Robinson N. Developing an acupuncture protocol for treating phantom limb pain: a Delphi consensus study. Acupunct Med. 2015;33(1):42-50.

15. Dworkin RH, Turk DC, Farrar JT, Haythornthwaite JA, Jensen MP, Katz NP, et al. Core outcome measures for chronic pain clinical trials: IMMPACT recommendations. Pain. 2005;113(1-2):9-19.

16. Katz J, Melzack R. The McGill Questionnaire. In: Turk DC, Melzack R, editors Handbook of pain assessment. 3rd ed. New York, London: The Guildford Press; 2011. p. 45-67.

17. O'Connor AB, Dworkin $\mathrm{RH}$. Assessment of pain and health related quality of life in chronic pain clinical trials. In: Turk DC, Melzack R, editors. Handbook of pain assessment. 3rd ed. New York: The Guilford Press; 2011. p. 474-88.

18. Snaith RP, Zigmond AS. The Hospital Anxiety and Depression Scale. 1st ed. London: GL Assessment; 1994. 
19. Cohen S, Williamson G. Perceived stress in a probability sample of the United States. In: Spacapam S, Oskamp S, editors. The social psychology of health. Newbury Park: Sage; 1988. p. 31-67.

20. Smith MT, Wegener ST. Measures of sleep. Arthritis Care Res. 2003;49(5S):S184-96.

21. Hurst $\mathrm{H}$, Bolton J. Assessing the clinical significance of change scores recorded on subjective outcome measures. J Manipulative Physiol Ther. 2004;27(1):26-35

22. Hertzog MA. Considerations in determining sample size for pilot studies. Res Nurs Health. 2008;31:180-91.

23. Hanley MA, Jensen MP, Ehde DM, Robinson LR, Cardenas DD, Turner JA, et al. Clinically significant change in pain intensity ratings in persons with spinal cord injury or amputation. Clin J Pain. 2006;22(1):25-31.

24. Field A. Discovering statistics using IBM SPSS statistics. 4th ed. Los Angeles: Sage; 2013.p.357-91.

25. Ritchie J, Spencer L, O'Connor W. Carrying out qualitative analysis. In: Ritchie J, Lewis J, editors. Qualitative research practice. London: Sage; 2003.p.21962.

26. Creswell JW, Plano Clark VL. Designing and conducting mixed methods research. 2nd ed. London: Sage; 2011.p.203-49.

27. Kadam P, Bhalerao S. Sample size calculation. Int J Ayurveda Res. 2010;1(1): 55-7.

28. McDonald AM, Knight RC, Campbell MK, Entwistle VA, Grant AM, Cook J, et al. What influences recruitment to randomised controlled trials? A review of trials funded by two UK funding agencies. Trials. 2006;7(9): doi:10.1186/ 1745-6215-7-9.

29. Adams M, Caffrey L, McKevitt C. Barriers and opportunities for enhancing patient recruitment and retention in clinical research: findings from an interview study in an NHS academic health science centre. Health Res Policy Syst. 2015; doi:10.1186/1478-4505-13-8.

30. Davidson JH, Khor KE, Jones LE. A cross-sectional study of post-amputation pain in upper and lower limb amputees, experience of a tertiary referral amputee clinic. Disabil Rehabil. 2010;32(22):1855-62.

31. Ehde DM, Czemiecki JM, Smith DG, Campbell KM, Edwards WT, Jensen MP et al. Chronic phantom sensations, phantom pain, residual limb pain, and other regional pain after lower limb amputation. Arch Phys Med Rehabil. 2000;81(8):1039-44.

32. Hondras MA, Long CR, Haan AG, Spencer LB, Meeker WC. Recruitment and enrolment for the simultaneous conduct of 2 randomized controlled trials for patients with subacute and chronic low back pain at a CAM research center. J Altern Complement Med. 2008;14(8):983-92.

33. Robinson L, Czerniecki J, Ehde D, Edwards T, Judish D, Goldberg M, et al Trial of amitriptyline for relief of pain in amputees: results of a randomized controlled study. Arch Phys Med Rehabil. 2004;85(1):1-6.

34. Karanicolas PJ, Farrokhyar F, Bhandari M. Practical tips for surgical research: blinding: who, what, when, why, how? Can J Surg. 2010;53(5):345-8.

35. Farrar JT, Young Jr JP, LaMoreaux L, Werth JL, Poole RM. Clinical importance of changes in chronic pain intensity measured on an 11-point numerical pain rating scale. Pain. 2001;94(2):149-58.

36. Thompson BT, Schoenfeld D. Usual care as the control group in clinical trials of nonpharmacologic interventions. Proc Am Thorac Soc. 2007;4(7):577-82.

37. Chan AW, Tetzlaff JM, Gotzsche PC, Altman DG, Mann H, Berlin JA, et al. SPIRIT 2013 explanation and elaboration: guidance for protocols of clinical trials. BMJ. 2013;346: doi: 10.1136/bmj.e7586.

38. Robiner WN. Enhancing adherence in clinical research. Contemp Clin Trials. 2005:26(1):59-77.

39. Smith SM, Wang AT, Katz NP, McDermott MP, Burke LB, Coplan P, et al. Adverse event assessment, analysis, and reporting in recent published analgesic clinical trials: ACTTION systematic review and recommendations. Pain. 2013;154(7):997-1008.

40. Ioannidis JP, Evans SJ, Gotzsche PC, O’Neill RT, Altman DG, Schulz K, et al. Better reporting of harms in randomized trials: an extension of the CONSORT statement. Ann Intern Med. 2004;141(10):781-8.

41. Cruccu G, Sommer C, Anand P, Attal N, Baron R, Garcia-Larrea L, et al. EFNS guidelines on neuropathic pain assessment: revised 2009. Eur J Neurol. 2010;17(8):1010-18.

42. Haanpää M, Attal N, Backonja M, Baron R, Bennett M, Bouhassira D, et al NeuPSIG guidelines on neuropathic pain assessment. Pain. 2011;152(1):14-27.

43. Bower P, Brueton V, Gamble C, Treweek S, Smith CT, Young B, et al. Interventions to improve recruitment and retention in clinical trials: a survey and workshop to assess current practice and future priorities. Trials. 2014; 15(399): doi:10.1186/1745-6215-15-399.
44. Edwards PJ, Roberts I, Clarke MJ, Diguiseppi C, Wentz R, Kwan I, et al. Methods to increase response to postal and electronic questionnaires. Cochrane Database Syst Rev. 2009;(3):MR000008: doi: 10.1002/14651858. MR000008.pub4.

45. Fortington LV, Geertzen JH, van Netten JJ, Postema K, Rommers GM, Dijkstra PU. Short and long term mortality rates after a lower limb amputation. Eur J Vasc Endovasc Surg. 2013;46(1):124-31.

46. Wampold BE, Serlin RC. The consequence of ignoring a nested factor on measures of effect size in analysis of variance. Psychol Methods. 2000; 5(4):425-33

\section{Submit your next manuscript to BioMed Central and we will help you at every step:}

- We accept pre-submission inquiries

- Our selector tool helps you to find the most relevant journal

- We provide round the clock customer support

- Convenient online submission

- Thorough peer review

- Inclusion in PubMed and all major indexing services

- Maximum visibility for your research

Submit your manuscript at www.biomedcentral.com/submit
C Biomed Central 